\title{
L'accompagnement en formation chez les éducateurs à l'environnement : À la recherche d'une identité professionnelle
}

Maryline Lair

\section{OpenEdition}

Journals

Édition électronique

URL : http://journals.openedition.org/ere/378

DOI : $10.4000 /$ ere.378

ISSN : 2561-2271

Éditeur

Centr'ERE

Édition imprimée

Date de publication : 1 septembre 2016

ISSN : 1373-9689

\section{Référence électronique}

Maryline Lair, « L'accompagnement en formation chez les éducateurs à l'environnement : À la

recherche d'une identité professionnelle », Éducation relative à l'environnement [En ligne], Volume 13 - 1 I

2016, mis en ligne le 15 septembre 2016, consulté le 21 février 2020. URL : http://

journals.openedition.org/ere/378; DOI : 10.4000/ere.378 


\title{
L'accompagnement en formation chez les éducateurs à l'environnement : À la recherche d'une identité professionnelle
}

\author{
Maryline Lair
}

1 Mon expérience d'animatrice de réseaux d'éducation à l'environnement (en Île-deFrance, puis en Bretagne), m'a conduite à travailler avec de nombreux éducateurs dans ce domaine. Parce qu'il s'investit dans un métier relationnel et parce qu'il juge les enjeux de l'éducation à l'environnement importants voire impératifs, l'éducateur à l'environnement est souvent traversé par des doutes, des remises en question sur son métier, son rôle et son efficacité. Pour que l'éducation à l'environnement contribue pleinement à la construction d'une identité écocitoyenne, la formation des éducateurs est un facteur essentiel. Or la formation de formateurs en éducation au développement durable de l'École supérieure du professorat et de l'éducation (ESPE) de l'Université de Clermont-Ferrand m'a donné l'occasion de questionner plus spécifiquement le lien générationnel entre les éducateurs à l'environnement. J'ai ainsi voulu comprendre ce que pouvait être ce lien et les temps de co-formation entre professionnels de différentes générations, et comment ils pouvaient contribuer à la professionnalisation des acteurs en favorisant le développement de nouvelles compétences et la construction d'une identité professionnelle en tant qu'éducateurs à l'environnement. Ce travail a également questionné la place des réseaux français associatifs d'éducation à l'environnement dans l'accompagnement en formation et la co-formation des professionnels. 


\section{Les éducateurs à l'environnement salariés du secteur associatif}

2 En France, les associations sont un des piliers de l'éducation à l'environnement. Ceci a notamment été favorisé par des dispositifs d'emplois. Comme le souligne un rapport coordonné par le Centre de ressources du dispositif local d'accompagnement en environnement (CRDLA Environnement) ${ }^{1}$ et le Réseau École et Nature (2008), sur un état des lieux de l'activité en éducation à l'environnement et au développement durable (EEDD),

[...] dans les années 90, ces associations se sont appuyées sur les dispositifs d'aide à l'emploi et à l'insertion. Puis c'est l'apparition du dispositif Nouveaux Services Emplois-Jeunes (NSEJ) ${ }^{2}$ qui a largement contribué au développement rapide de l'emploi dans le secteur : $22 \%$ des emplois-jeunes en environnement ont été créés dans le secteur éducatif. Ces associations d'éducation à l'environnement, aux histoires et parcours divers, se sont professionnalisées au fil des décennies, dans leurs organisations et modes de gouvernance au sein de leurs équipes salariées, et en développant de nouvelles activités; on peut noter aussi "une montée en puissance de l'expertise associative » (Aspe et Jacqué, 2012).

3 Cette professionnalisation des associations a été nécessaire pour exister et défendre leur place (Wittorski, 2008). Aujourd'hui, les associations embauchent une part importante des jeunes formés en éducation à l'environnement.

L'éducation à l'environnement représenterait entre 3000 et 4000 emplois en France, dans le secteur associatif (Cabinet Ithaque, 2010). Des données nationales provenant du Tableau de bord de l'Éducation à l'Environnement et au Développement Durable (outil interne au Réseau École et Nature) montrent que les éducateurs à l'environnement sont pour la plupart assez jeunes et ont un haut niveau de formation (une majorité détient un diplôme de niveau III au moins).

5 L'éducateur à l'environnement, derrière une appellation à peu près consensuelle, correspond toutefois à une diversité de professionnels et de métiers, celle-ci étant accrue par les évolutions de l'éducation à l'environnement (évolution du secteur associatif, évolution des pratiques, évolution des publics, etc.). Si les parcours de formation sont très variables d'un professionnel à l'autre (pour des raisons diverses : vocation, ancienneté, motivation, etc.), la construction identitaire l'est tout autant. Si, au sens de Wittorski (2008), on considère la professionnalisation comme impliquant un double mouvement d'acquisition de savoirs et de compétences professionnelles «en situation réelle», et de construction d'une identité, alors il nous semble que les processus de professionnalisation des éducateurs à l'environnement restent fragiles.

6 À cet effet, se pose la question suivante : dans le processus de formation permanente d'un éducateur à l'environnement, faut-il privilégier la transmission entre pairs et entre générations d'éducateurs à l'environnement pour participer à la construction d'une identité professionnelle, et ainsi favoriser une professionnalisation assumée de l'éducateur à l'environnement? 


\section{L'accompagnement en formation et l'accompagnement intergénérationnel}

7 Dans son travail sur le sens des mots et le sens que l'on donne à l'accompagnement, Paul (2004) propose une définition minimale de toute forme d'accompagnement comme: "être avec et aller vers, sur la base d'une valeur symbolique, celle du partage ». Les finalités des diverses pratiques d'accompagnement (conseil, mentorat, tutorat, compagnonnage et autre) sont hétérogènes : résolution de situation-problème, recherche d'efficacité, autonomisation de la personne, recherche de sens, etc. Elles ne concernent plus aujourd'hui uniquement un duo « ancien - novice ", mais se centrent sur la relation partenariale et son cheminement. La posture de l'accompagné relève alors d'un mouvement de réciprocité, dans le sens où il est un accompagnateur en devenir.

8 Pineau (2002) parle lui de l'accompagnement en formation comme d'un processus entre "solidarité humaine » et "professionnalisation », où il y a création de liens interpersonnels.

9 Mener un travail sur l'accompagnement et la co-formation c'est prendre en compte le temps, le lieu et l'autre :

- Le temps / la temporalité: le lien générationnel et les relations d'accompagnement impliquent un double processus temporel, à la fois ici et maintenant entre deux individus (soi et l'autre), mais également entre soi, à différentes échelles de temps (hier, aujourd'hui et demain). Penser la relation d'accompagnement, c'est s'inscrire dans le temps (Paul, 2004).

- Le lieu / l'espace: L'accompagnement demande du temps et demande aussi à être matérialisé : s'il existe, il doit être nommé, dit, localisé. Lui donner un espace c'est le reconnaître et reconnaître les acteurs en présence (Paul, 2004).

- L'altérité / l'altération: Les relations d'accompagnement sont une rencontre de soi, des autres et du monde (Paul, 2004). L'appartenance à un groupe social et la construction d'une identité professionnelle sont permises dans un mouvement de confrontation, avec des allers et des retours, et dans un mouvement d'altération ou pour le dire autrement « différence par changement», selon le Dictionnaire historique de la langue française (2012). Cette reconnaissance de l'autre favorise ainsi le développement professionnel, par transformations individuelles et collectives de "composantes identitaires mobilisées ou pouvant être mobilisées dans des situations professionnelles » (Barbier, Chaix, et Demailly, 1994).

10 Nous pouvons toutefois nous interroger sur la pertinence de l'accompagnement entre pairs, et d'autant plus entre éducateurs à l'environnement de générations différentes, quand le champ de l'éducation à l'environnement est bousculé par le développement durable et l'éducation au développement durable, quand le secteur associatif connaît une crise importante (Tchernonog, 2013), où tout simplement quand l'éducateur d'hier n'est plus celui d'aujourd'hui (Aspe et Jacqué, 2012)? D'une transmission, de $l^{\prime}$ « ancien » au « jeune » sur des savoirs stables, nous passons à une relation entre pairs, où les plus anciens ont autant à apprendre que les plus jeunes (Paul, 2009). 


\section{Le cas des éducateurs du Réseau breton d'éducation à l'environnement}

11 Le REEB est une association-réseau régionale rassemblant 130 personnes morales et une trentaine d'adhérents à titre individuel. Les structures adhérant au REEB ont pour point commun de faire de l'éducation à l'environnement en Bretagne. Toutes les structures adhérentes au réseau n'emploient pas des éducateurs à l'environnement ; certaines associations n'ont pas de salariés, d'autres n'ont pas de salarié sur un poste d'éducateur à l'environnement.

Les enquêtes menées depuis 20 ans auprès des acteurs de l'éducation à l'environnement en Bretagne n'ont pas ou peu fait la distinction entre salariés du secteur associatif ou salariés des collectivités publiques ${ }^{3}$. De plus, elles n'ont pas ou peu fait également la distinction entre professionnels faisant de l'éducation à l'environnement leur métier, et professionnels faisant dans leur métier de l'éducation à l'environnement. Nous n'avons donc pas beaucoup de données préalables sur les éducateurs à l'environnement du secteur associatif en Bretagne.

13 Pour recueillir des données sur les représentations de la formation, sur les attentes visà-vis des pairs, les apports des relations d'accompagnement, etc., j'ai identifié 130 éducateurs à l'environnement travaillant au sein des associations adhérentes au REEB, qui ont ainsi reçu un questionnaire papier comportant 10 questions. Au total, 33 personnes l'ont retourné.

14 Dans notre échantillon, l'âge est très corrélé à l'ancienneté en éducation à l'environnement. Les individus se répartissent de façon assez régulière de 23 à 62 ans.

15 Une série de questions concernait le parcours de formation des répondants. À la question "Comment définiriez-vous votre parcours de formation? ", la majorité des répondants font référence à la fois à leur formation initiale et à leur environnement. Il est moins fait référence à la formation continue; on observe que ce sont surtout les plus jeunes qui n'en parlent pas : cela peut s'expliquer par le fait qu'ils sortent tout juste de leur parcours en formation initiale.

16 Deux questions ouvertes ont permis d'identifier le sens donné par les enquêtés aux relations d'accompagnement: "Dans votre parcours de formation, avez-vous, entre autres, été formé par vos pairs?» et «De la même façon, vous est-il arrivé d'accompagner des jeunes professionnels ou des étudiants et étudiantes en éducation à l'environnement?». La grande majorité des répondants ont été formés par leurs pairs et ont également accompagné des plus jeunes dans leur professionnalisation.

17 Face à une difficulté et en recherche d'une résolution («Lorsque que vous rencontrez une difficulté dans votre pratique professionnelle, que faites-vous ? »), les enquêtés ont largement mis en avant deux types de réponses : cheminer par soi-même (démarche d'autoformation, autonomie dans la résolution d'un problème, selon son propre rythme et en choisissant ses ressources) et faire appel à des collègues de travail proches d'eux. Les enquêtés privilégient un soutien auprès d'individus et de ressources qu'ils choisissent ; très rarement, ils se tournent vers des organisations, même de proximité comme leur conseil d'administration, ou le réseau régional d'éducation à l'environnement.

18 À la question «Que pourriez-vous attendre d'un accompagnement avec une personne éducatrice à l'environnement plus expérimentée?» la réponse la plus souvent 
exprimée est "analyser ma pratique professionnelle». Aucun des 33 répondants n'a estimé qu'il n'avait rien à attendre d'un accompagnement avec un éducateur à l'environnement plus expérimenté.

19 Enfin, à la question "Quelle serait votre démarche si vous deviez accompagner un éducateur ou une éducatrice à l'environnement plus jeune que vous? », la réponse la plus souvent exprimée est «transmettre mon expérience». Ce sont les réponses liées au développement des compétences qui sont le moins associées aux relations d'accompagnement.

L'ensemble des résultats nous permettent d'observer des fonctionnements communs ou différents selon les générations d'éducateurs à l'environnement :

- Pour résoudre une difficulté professionnelle, les répondants privilégient un rapport aux pairs ou autres personnes composant leur réseau professionnel plutôt qu'aux organisations (association, réseau).

- Les résultats montrent un lien plus important entre les plus jeunes éducateurs à l'environnement (de 20 à 35 ans) et les plus anciens (après 45 ans). Les plus jeunes comme les plus anciens seraient plus à la recherche de l'autre, en attente d'un échange sur leur métier et sur l'éducation à l'environnement.

- Les éducateurs à l'environnement entre 35 et 45 ans semblent être plus orientés vers soi et en questionnement dans une démarche réflexive de leur pratique professionnelle.

\section{Discussion}

21 Cette enquête a été menée pour vérifier l'intérêt et les apports des relations d'accompagnement entre jeunes éducateurs à l'environnement et éducateurs à l'environnement "expérimentés », dans une visée de développement professionnel des uns et des autres. Elle a confirmé, comme le disait Pineau (2002), qu'il n'est pas toujours facile d'identifier des relations d'accompagnement; elles sont « toujours là et pourtant pas simple à caractériser » tel un territoire labile.

22 Rendre possible l'accompagnement tout au long de la trajectoire professionnelle, c'est laisser les relations d'accompagnement s'exprimer dès la formation initiale, puis en cours de carrière. C'est également tenir compte du fait que chaque âge a ses particularités et ses propres attentes vis-à-vis des relations d'accompagnement.

23 Selon notre enquête, il semble que le jeune éducateur à l'environnement ait un rapport fort au développement d'un savoir-être relationnel et d'une relation moi-milieu (Riverin-Simard, 1998), le milieu étant ici représenté par les personnes en place dans le milieu (les autres) plutôt que par les organisations.

24 Ensuite arriverait une étape où l'éducateur à l'environnement entre dans une démarche réflexive sur sa pratique (Schön, 1994), étape nécessaire pour transformer son expérience en ressource, pour soi et pour les autres. Cette étape profitera d'un accompagnement par un formateur capable d'encadrer des méthodes d'analyse des pratiques. Puis, l'éducateur à l'environnement plus expérimenté prioriserait à nouveau les relations interpersonnelles.

25 L'éducateur à l'environnement se construit une représentation du métier et du champ de l'éducation à l'environnement en interaction avec l'autre, "dans un espace d'intersubjectivité construit par les personnes en présence » (Vinatier, 2002), dans un mouvement de réciprocité. Favoriser les relations d'accompagnement entre pairs ne 
reviendrait alors pas à transformer une démarche spontanée où les acteurs se choisissent, en formalisant un processus (Paul, 2004), mais en créant les conditions favorables à la reconnaissance d'un temps et d'un lieu de rencontres.

C'est peut-être le défi d'un réseau d'éducation à l'environnement que de mieux favoriser ces espaces et ces temps de reconnaissance. Des travaux de recherche ont été menés sur les réseaux, par le mouvement des Réseaux d'échanges réciproques de savoirs (RERS) et notamment par Héber-Suffrin qui questionnait cette organisation sociale «en réseau»: "ce qu'il nous faut, ce sont des structures qui mettent les [personnes] en rapport les unes avec les autres et permettent, par là, à chacun, de se définir en apprenant et en contribuant à l'apprentissage d'autrui. " (Illich, cité par Héber-Suffrin, 2000). La relation mentorale est, parmi les différentes formes d'accompagnement, une relation où l'on se choisit, une relation de réciprocité et de solidarité (Paul, 2004) : elle se définit entre une personne d'expérience et une personne moins expérimentée. Cette relation d'aide permet aux uns (les mentors) de réaménager leur rapport à eux-mêmes, aux autres et au monde, et aux autres (les "novices ») d'apprendre, dans cette phase de transition que constitue l'entrée dans la vie active, les ressorts de cette triple relation (à soi, aux autres et au monde) qui permet d'harmoniser l'orientation professionnelle et le projet de vie.

\section{Conclusion}

L'idée générale qui a sous-tendu cette recherche est qu'il est plus facile de répondre ensemble aux questions de sens que chacun se pose et à la quête de sens que chacun mène. Dans le regard de l'autre peut émerger des réponses personnelles.

Notre vision de la formation concerne l'être tout entier : «la formation est plus qu'un rapport au savoir, elle est rapport au monde, elle est une construction de l'être-aumonde » (Cottereau, 2001). Notre démarche a donc été de penser la formation aussi à travers les relations d'accompagnement, pour voir en quoi elles pouvaient participer à la construction identitaire des éducateurs à l'environnement et développer des compétences et capacités nouvelles (par la co-formation notamment).

Selon Paul (2009), l'accompagnement répond à une triple visée : la place du sujet dans son groupe social et sa culture d'appartenance, la construction d'une identité singulière et l'insertion dans l'ordre de l'humain. Rappelons que l'appartenance à un groupe social et la construction d'une identité professionnelle sont permises dans un mouvement de confrontation, avec des allers et des retours (confrontation avec l'autre, avec soi et avec l'environnement), et dans un mouvement d'altération (« différence par changement »).

À l'image des réseaux d'échanges réciproques de savoirs, les réseaux d'éducation à l'environnement sont des organisations «apprenantes" qui gagneraient à travailler davantage sur le développement de la capacité d'autoformation des individus, en favorisant une démarche de réciprocité avec les pairs (Héber-Suffrin, 2001). Le rôle des réseaux d'éducation à l'environnement est à la fois de créer les conditions favorables au développement des relations d'accompagnement, de développer de nouvelles formes de formations et d'apprentissages telles la co-formation, de développer l'apprenance des éducateurs à l'environnement, et de favoriser l'émergence de nouveaux formateurs en éducation à l'environnement. 


\section{BIBLIOGRAPHIE}

Aspe, C. et Jacqué, M. (2012). Environnement et société. Paris: La Maison des sciences et de l'homme.

Barbier, J.-M., Chaix, M.-L. et Demailly, L. (1994). Recherche et développement professionnel : Matériaux pour une étude de la place de la recherche dans les processus de professionnalisation. Recherche et Formation, 17, 5-8.

Cabinet Ithaque (2010). Étude sur l'éducation à l'environnement vers le développement durable dans les métiers de l'animation. Paris: Ministère des Sports. Centre de ressources du dispositif local d'accompagnement en environnement et Réseau École et Nature (2008). Éducation à l'environnement vers un développement durable : État des lieux, activités, économie et emploi. Paris: Avise.

Cottereau, D. (2001). Pour une formation écologique. Complémentarité des logiques de formation. Éducation Permanente, 148, 57-67.

Héber-Suffrin, C. (2000). Réseaux en mouvement. Éducation Permanente, 144, 99-118.

Héber-Suffrin, C. (2001). Échangeons nos savoirs ! Paris: La Découverte.

Paul, M. (2004). L'accompagnement : une posture professionnelle spécifique. Paris: L'Harmattan. Paul, M. (2009). Accompagnement. Recherche et formation, 62, 91-107.

Pineau, G. (2002). L'accompagnement en formation : de l'avant-naissance à l'après-mort, Entre solidarité et professionnalité. Éducation Permanente, 153, 29-41.

Rey, A. (2012). Dictionnaire historique de la langue française. Paris: Dictionnaires Le Robert. Riverin-Simard, D. (1998). Histoire de vie professionnelle d'accompagnateurs. Dans Pineau, G. Accompagnement et histoire de vie (p.157-173). Paris: L'Harmattan.

Schön, D.-A. (1994). Le praticien réflexif. À la recherche du savoir caché dans l'agir professionnel. Montréal, Québec : Éditions Logiques.

Tchernonog, V. (2013). Le paysage associatif français. Mesure et évolutions. Paris: Dalloz Juris Associations.

Vinatier, I. (2002). La construction de l'identité professionnelle en acte dans la relation de service. Éducation Permanente, 151, 11-27.

Wittorski, R. (2008). La professionnalisation. Savoirs, 17, 9-36. DOI : 10.3917/savo.017.0009.

\section{NOTES DE FIN}

1. Le Dispositif local d'accompagnement (DLA) est un dispositif d'accompagnement au développement et à la consolidation des structures d'utilité sociale sur les territoires, créé en 2002 par l'État français et la Caisse des dépôts et consignations.

2. Le dispositif NSEJ, dit "emplois-jeunes ", a été appliqué entre 1997 et 2002 ; c'est un type de contrat de travail de droit privé français, rentrant dans la catégorie des contrats aidés.

3. On se réfère à plusieurs études et diagnostics coordonnés entre autres par le REEB :

- 1998, Diagnostic de l'éducation à l'environnement en Bretagne, Évaluation du poids socio-économique, coordonné par le Collectif breton en vue de la préparation des Assises régionales de l'EE (1999); 
- 2001, Emploi en EEDD en Bretagne, coordonné par le REEB ;

- 2007, Recensement des besoins de formation des éducateurs à l'environnement, coordonné par le REEB (réalisation Dominique Cottereau) ;

- 2012, Étude emploi/formation en EEDD, en Région Bretagne, coordonnée par le REEB.

\section{INDEX}

Mots-clés : éducation à l'environnement, formation, identité professionnelle, accompagnement, milieu associatif, co-formation, dynamique intergénérationnelle

Keywords : environmental education, training, professional identity, accompaniment, associative environment, co-training, intergenerational dynamics

\section{AUTEUR}

\section{MARYLINE LAIR}

Maryline Lair est animatrice et coordinatrice de réseaux d'éducation à l'environnement depuis 2002, et plus spécifiquement, au sein du Réseau d'éducation à l'environnement en Bretagne depuis 2004. Cet article est le fruit d'un projet de recherche mené dans le cadre du Master 2 Formation de formateurs en éducation au développement durable à l'Université Blaise Pascal à Clermont-Ferrand, et accompagné par Jean-Marc Lange (directeur de mémoire). 